\title{
Epilepsy Genetics Revolutionizes Clinical Practice
}

\author{
Ingrid E. Scheffer ${ }^{1,2}$ \\ ${ }^{1}$ Department of Medicine, Florey Institute of Neurosciences \\ and Mental Health, University of Melbourne, Austin Health, \\ Melbourne, Australia \\ 2 Department of Paediatrics, University of Melbourne, Royal Children's \\ Hospital, Melbourne, Australia
}

\begin{abstract}
Address for correspondence Ingrid E. Scheffer, MBBS, PhD, FRACP, Austin Health, Melbourne Brain Centre, 245 Burgundy Street, Heidelberg, Victoria 3084, Australia (e-mail: scheffer@unimelb.edu.au).
\end{abstract}

Neuropediatrics 2014;45:70-74.

\begin{abstract}
Keywords

- epilepsy

- genetics

- epilepsy syndromes

- DEPDC5

- epileptic encephalopathies

Objectives Epilepsy genetics has undergone a revolution in the past 19 years since the discovery of the first gene for epilepsy. The story of our increasing knowledge and how it impacts on patient care is presented with reference to recent discoveries. Understanding the significance of a genetic variant is challenging both in terms of molecular pathogenicity and in how this finding fits into the rubric of causation. In some cases, it may only be a contributing susceptibility factor; whereas in others, it explains the patient's disease.

Methods A brief overview of the clinicomolecular approaches is discussed in the context of the discovery of epilepsy genes. These include family studies and, more recently, next generation sequencing using multigene panels and whole exome sequencing.

Results Recent studies illustrating the way in which epilepsy genetics is changing clinical practice are described. A particular focus is DEPDC5, the first gene for nonlesional focal epilepsy likely to be relevant to sporadic patients with focal epilepsies and those from small families, in contrast to rare large families with autosomal dominant focal epilepsies. As DEPDC5 is a negative regulator of the mammalian target of rapamycin (mTOR) pathway, it is likely that some patients with DEPDC5 mutations may have malformations of cortical development akin to the two-hit hypothesis suggested in tuberous sclerosis. The greatest impact of epilepsy genetics at a clinical level is for patients with epileptic encephalopathies as many have de novo mutations-a rapidly expanding list of causative genes is being found.

Conclusion Epilepsy genetics is changing clinical practice enabling diagnosis in many patients, informing our understanding of comorbidities, prognosis, and genetic counseling. Importantly, a genetic finding may impact on treatment choices. At a biological level, new insights promise to lead to the development of novel therapies and bring together the seemingly disparate genetics of nonlesional epilepsies and epilepsies associated with cortical malformations.
\end{abstract}

\section{Introduction}

It was not that long ago that the search for epilepsy genes was considered futile. Indeed, when I embarked on my $\mathrm{PhD}$ in epilepsy genetics in 1991, a luminary in the field of neurogenetics laughed at the prospect of making scientific inroads into this complex field. To his credit, 15 years later he admitted that his view was wrong, but it did indeed reflect the view of clinicians and scientists of the time. Now, I am delighted to see that genetics permeates all areas of pediatric neurology and is revolutionizing the way we evaluate and manage patients with epilepsy. published online March 10, 2014 (c) 2014 Georg Thieme Verlag KG Stuttgart · New York
DOI http://dx.doi.org/ 10.1055/s-0034-1371508. ISSN 0174-304X. 
The cornerstone of recent success in epilepsy genetics has been careful electroclinical phenotyping coupled with major advances in molecular techniques. At no time has it been more exciting than now with gene discovery occurring at a cracking pace. Finding the genetic mutation is challenging and it takes significant expertise to identify which variant is causal with the enormous data sets obtained using massively parallel sequencing technology. However, the translation back to clinical care lies in the hands of the pediatric neurologist who has a critical role in interpreting whether a genetic variant identified is causal for the child's phenotype and in taking the clinical science forward. In many ways, the pediatric neurologist's expertise has never had more relevance as they have a key role in identification of comorbidities, favorable and adverse treatment responses, prognostic indicators, and in ensuring timely genetic counseling. Even more challenging is the translation back to the clinic of novel therapeutic approaches tested in animal and in vitro models. Targeted genetic therapies hold the promise of dramatically improving outcomes both in terms of seizure control and cognition and, one day I hope, in preventing disease.

\section{Overview of Epilepsy Genetics}

The genetics of the epilepsies like other common diseases, such as hypertension and asthma, frequently follow complex inheritance. Thus, most individuals with genetically determined epilepsy are thought to have a polygenic basis in which multiple genes of low-to-moderate risk interact, sometimes with an environmental contribution, to produce the epileptic disease. At present, relatively little is known of these susceptibility alleles although rare variants in ion channel genes (e.g., the calcium channel subunit gene $C A C N A 1 H$ ) have been reported. ${ }^{1,2}$ In addition, copy number variants contribute as risk alleles to the genetic generalized epilepsies (GGEs), the group that forms the majority of genetic epilepsies and accounts for one-third of all epilepsies. Three copy number variants, $15 q 13.3,15 q 11.2$, and $16 \mathrm{p} 13.11$, are each found in approximately $1 \%$ of GGEs. ${ }^{3-5}$

Although many of the common epilepsies are thought to follow complex inheritance, there is increasing evidence that de novo mutations in genes of major effect account for a significant number of cases. ${ }^{6}$ A recent example is the finding that de novo SLC2A1 mutations, which lead to glucose transporter 1 deficiency, cause GGEs with in vitro studies showing major transporter dysfunction. ${ }^{7}$ Previously, it was well recognized that glucose transporter 1 encephalopathy, in which the patient has severe neurological features of intellectual disability, epilepsy, movement disorders, and often microcephaly, was frequently due to a de novo SLC2A1 mutation, but it was not recognized that milder epilepsies could also be due to a de novo mutation. ${ }^{8}$ We recently hypothesized that the markedly different functional effects demonstrated by different SCL2A1 mutations may mean that mutations causing major dysfunction are consistent with dominant inheritance while those causing minimal dysfunction contribute as susceptibility alleles. ${ }^{7}$ This work requires confirmation but introduces a multi-layered model of considering the contribution a specific gene may make to the inheritance patterns observed in the epilepsies.

Monogenic epilepsies were first identified through family studies in rare large multiplex families with dominant focal or generalized epilepsies. ${ }^{9,10}$ Many ion channels have been implicated and include a range of sodium, potassium, and calcium voltage-gated ion channel subunit genes as well as ligand-gated ion channels such as nicotinic and $\gamma$-aminobutyric acid receptor subunits. ${ }^{11}$ More recently, the importance of de novo mutations of dominant genes has been appreciated as a major cause of the severe group of epileptic encephalopathies (EE) with large scale international research efforts making major inroads into understanding the causative genes (see the section "Epileptic Encephalopathies"). While there is early evidence that de novo mutations account for sporadic cases of more common phenotypes, we are yet to fully understand the proportion of the common epilepsies that are due to de novo mutations.

The contribution of genetics to the etiology of the epilepsies can be conceptualized as a spectrum with monogenic epilepsies at one end. The largest part of the spectrum, in the middle, accounts for the major group of epilepsies that follow complex or polygenic inheritance and, at the other end of the spectrum, lies the acquired epilepsies. Acquired epilepsies are often structural in nature and may be due to vascular, neoplastic, traumatic, immune, and infectious causes.

\section{Molecular Genetic Approaches}

Family studies formed the early substrate of successful molecular genetic research in the epilepsies and led to the discovery of the first gene in $1995^{\circ}$ and further gene identification over the following 15 years. ${ }^{11}$ Some might argue that the "low hanging fruit" has been picked and there is little to gain from further endeavors focusing on family studies. Recent successes in gene discovery (see the section "Genetic Focal Epilepsies") highlight that this sentiment is incorrect and family studies can still play a key role. Massively parallel sequencing, using whole exome, whole genome, or multigene panel approaches, can be applied to large or small families or sporadic cases to identify genes. This allows us to interrogate whether specific genes are relevant to a broader population of individuals with epilepsy in a cost-effective manner.

\section{Genetic Focal Epilepsies}

\section{Autosomal Dominant Nocturnal Frontal Lobe Epilepsies}

Since we described the first genetic focal epilepsy in 1994, several rare familial focal epilepsies have been reported. ${ }^{12}$ The first gene for epilepsy was identified in 1995 in autosomal dominant nocturnal frontal lobe epilepsy (ADNFLE), and now three neuronal nicotinic acetylcholine receptor subunits have been implicated. ${ }^{9,13,14}$ However, overall, the molecular basis has been solved for fewer than $20 \%$ of families with ADNFLE.

ADNFLE is a distinctive clinical disorder characterized by clusters of nocturnal motor seizures, with a median age of onset of 8 years. Patients typically have eight seizures per 
night over a few hours. Approximately, 70\% of patients experience a nonspecific aura on awakening prior to their motor attack. The motor features may be tonic or hyperkinetic and thrashing in nature. The electroencephalogram (EEG) is unhelpful because it may be obscured by movement artifact or may even appear normal. Misdiagnosis in this disorder is common, with some individuals regarding their seizures as normal sleep, others being diagnosed with parasomnias and some even thought to be hysterical because of retained awareness during the attacks. ADNFLE follows autosomal dominant inheritance with approximately $70 \%$ penetrance. ${ }^{15}$

We recognized a more severe form of ADNFLE in $2008 .^{16}$ In this disease, some family members have psychiatric disorders and intellectual disability together with highly refractory epilepsy. Recently, we mapped a large Australian family to chromosome 9q34.3 with a logarithm of the odds score of 2.71. This encompassed a $2.63 \mathrm{Mb}$ region that included 99 genes. Whole exome sequencing identified a missense mutation in the sodium-activated potassium channel gene KCNT1. ${ }^{17}$ This missense variant segregated perfectly with affected individuals and was not found in controls. Following identification of the mutation in the family with severe ADNFLE, a further 108 cases with nocturnal frontal lobe epilepsy, including those following dominant inheritance and sporadic cases, were screened for mutations in KCNT1. Three further mutations were identified with the majority of mutations located in the NAD+ binding site, which has a role in channel modulation of the KCNT1 protein. KCNT1 is highly expressed in brain and also called SLACK (sequenced like a calcium-activated potassium channel). SLACK binds with SLICK (KCNT2) to form heterotetrameric channels.

Four mutations were identified including a de novo change in a sporadic patient. ${ }^{17}$ Severe ADNFLE had an earlier mean onset of 6 years compared with 10 years in the classical form of ADNFLE. Intellectual disability was observed in two of the four families and psychiatric problems were noted in all four families, always occurring in concert with frontal lobe epilepsy. Psychiatric problems encompassed psychosis, catatonia, and aggression. Thus, there are now four genes implicated in ADNFLE with the more severe form being associated with psychosis and intellectual disability. Yet, in the majority of families, the causative gene is yet to be established.

\section{Familial Focal Epilepsy with Variable Foci}

We first identified the syndrome of familial focal epilepsy with variable foci (FFEVF) in 1998 in an Australian family in which three individuals had frontal lobe epilepsy, five had temporal lobe epilepsy, and one had parietal lobe epilepsy. ${ }^{18}$ The concept underlying this syndrome was of autosomal dominant inheritance of focal epilepsy that emanated from different cortical regions in different family members. Since 1998, two boys have been born into the original Australian family. Both have autism spectrum disorder and one has temporal lobe epilepsy and intellectual disability. One of the older members of the original family also had autism spectrum disorder and intellectual disability. This family was mapped in 2012 to chromosome 22q12 to the previously identified locus for FFEVF. ${ }^{19,20}$
Whole exome sequencing was performed with particular attention paid to the $22 \mathrm{q} 12$ locus encompassing a $5.3 \mathrm{Mb}$ region. A truncation mutation was identified in the gene DEPDC5. ${ }^{21}$ Mutations were found in seven of the eight reported families with FFEVF. ${ }^{21}$ In a large Dutch family and two Spanish families, truncation mutations were discovered. ${ }^{22-24}$ Three French-Canadian families had the same founder amino acid deletion mutation. ${ }^{23}$ The penetrance of FFEVF was quite low with a mean of $66 \%$ (range, $50-82 \%$ ).

DEPDC5 encodes disheveled, Egl-10, and Pleckstrin domain containing protein 5 which has orthologs found in diverse species including mosquitos. Mouse Depdc5 was studied with quantitative real-time polymerase chain reaction on RNA from various mouse tissues which showed that Depdc5 was detected in all brain regions studied. ${ }^{21}$ It was also found throughout development from midgestation embryonic head through to neonatal and adult mouse brain. Confocal images with double immunostaining of Depdc5 in adult mouse brain showed that Depdc5 was expressed in neurons, but not in nonneuronal cells such as astrocytes. Depdc5 was localized to the perinuclear region with little or no extension into neuronal processes. DEPDC5 was also found in neurospheres from human-induced pluripotent stem cells and, again, was shown to be present in neural precursors and neurons, but it was absent in astrocytes. ${ }^{21}$

DEPDC5 has recently been identified as having an important role as mTOR inhibitor. The MTOR pathway is a master regulator of cell growth. ${ }^{25}$ Bar-Peled et al described the GTPase-activating protein activity toward Rags (GATOR) complex which comprises eight proteins for GATOR and divided it into two subcomplexes GATOR1 and GATOR2. GATOR1 is made of DEPDC5, Nprl2, and Nprl3. ${ }^{26}$ The GATOR complex is required for regulation of the TORC1 pathway by amino acids. Amino acids are one of the cues to cell growth and act through the Rag guanosine triphosphatases (GTPases) to promote mTORC1 translation to the surface of the lysosome in order for activation to occur. Inhibition of GATOR1 subunits renders mTORC1 signaling resistant to amino acid deprivation and thus constitutive mTORC1 activation occurs, similar to the neurobiology of tuberous sclerosis.

As focal epilepsy accounts for $60 \%$ of all epilepsies, we then asked whether DEPDC5 caused common nonlesional focal epilepsy. We performed high resolution melt curve analysis to genotype 82 unrelated probands drawn from families with two or more individuals with nonlesional focal epilepsy. Mutations were found in 10 of $82(12 \%)$ cases. There were six truncation mutations and three missense mutations with one mutation found in two families. The majority of mutations encoded premature termination codons, consistent with haploinsufficiency. In the 10 families with mutations, very different patterns of focal epilepsy were observed, with some having purely temporal lobe epilepsy, others frontal lobe epilepsy or just nocturnal frontal lobe epilepsy, while others had combinations from different cortical regions. In one family, one of the four individuals with temporal lobe epilepsy had a de novo DEPDC5 mutation that was not found in her affected relatives. 
Analysis of 95 affected individuals with DEPDC5 mutations showed that FFEVF was associated with frontotemporal lobe epilepsy in $70 \%$ of cases. ${ }^{21}$ Focal epilepsy ranged in severity from readily treatable to refractory despite multiple antiepileptic drugs. ${ }^{18,20}$ One of the critical questions we raised is whether DEPDC5 mutations are responsible for autism spectrum disorders, other psychiatric manifestations, and intellectual disability seen in a few individuals in these families. For example, there is a boy in the original Australian family who has the familial DEPDC5 mutation and autism spectrum disorder but has not yet had seizures. Thus, DEPDC5 is essentially the FFEVF gene but, just as importantly, it is relevant to smaller families and likely sporadic cases of nonlesional focal epilepsy. A key biological question is why different focal epilepsies occur in different family members.

\section{Epileptic Encephalopathies}

The greatest success in unravelling the genetics of the epilepsies has been in the group of the EE where genetics has already changed clinical practice. These severe disorders typically begin in an infant or a child who develops multiple types of seizures, abundant epileptiform activity on EEG, and developmental slowing or regression. These patients often have severe disability and refractory seizures that may continue into adult life. They may have comorbidities such as autism spectrum disorders and motor features and also have an increased mortality risk. Magnetic resonance imaging is usually normal or may show nonspecific features such as diffuse atrophy. Increasingly, it is now recognized that for many of these patients, their disorder is due to a de novo dominant mutation or, more rarely, recessive or mitochondrial DNA mutation.

Recent large scale studies incorporating next generation sequencing of patients with EE have suggested that up to $30 \%$ of cases can be solved with current technologies. This includes the prototypic model of Dravet syndrome in which more than $80 \%$ of patients have mutations of the $\alpha 1$ subunit gene of the sodium channel, SCN1A. Many other genes for EEs are now well recognized including CDKL5, STXBP1, SCN2A, SCN8A, and $K C N Q 2 .{ }^{24}$ With the use of multigene panels incorporating targeted next generation sequencing of specific genes, new genes have recently been identified such as CHD2, GRIN2A, and SYNGAP1.27,28

An alternative approach has been the use of whole exome sequencing in trios that involves the study of the proband and both parents. The strategy in these studies is to look for a variant present in the proband that is not present in either parent. ${ }^{29}$ Variants then undergo analysis for pathogenicity. This takes into account the nature of the amino acid change, whether it is likely to have a functional effect and its absence in large population databases of genomic variation such as the exome variant server or dbGaP.

These discoveries are having a huge impact on the diagnosis of these patients with devastating disorders. In some countries such as the United States, it is becoming commonplace for clinicians to be able to order whole exome sequencing commercially in their patients. Variants that are found can be tested in their parents to see if they have arisen de novo. In some instances, the variant may be inherited and still causative; for instance, the parent may be mosaic and unaffected or mildly affected, as has been shown in SCN1A family studies. ${ }^{30}$ Alternatively, it may be that an inherited variant is contributing to the patient's disorder as one of several genes underpinning complex inheritance. In other instances, the variant may be an inherited innocent polymorphism and not causative. Other forms of inheritance should also be remembered such as recessive inheritance which is particularly likely in the setting of consanguinity although compound heterozygosity may occur with both inbred and outbred families. Mitochondrial inheritance should not be forgotten. In addition, Xlinked recessive and $\mathrm{X}$-linked dominant inheritance is important to remember as well as the more unusual inheritance pattern of X-linked inheritance with male sparing. This latter form of inheritance is typically seen in PCDH19 encephalopathy which occurs in girls, and in which, males are normal transmitting carriers. ${ }^{31}$

Molecular genetics has truly revolutionized our approach in understanding the genetics of the epilepsies and identifying the cause in patients with epilepsy. A molecular diagnosis enables the end of the "diagnostic journey" which has often been prolonged, involved a myriad of investigations and considerable pain and anxiety for patients and their families. Yet, in many ways, finding the gene for a patient is just the beginning of the answer. Once an epilepsy gene is discovered, the phenotypic spectrum emerges as increasing numbers of patients are identified, facilitating diagnosis in other patients. The gene and its resultant protein then undergo intense scientific scrutiny in in vitro and in vivo models allowing us to develop an understanding of the biology of the seizure disorder and associated comorbidities. The most exciting and challenging aspect is the development of targeted therapeutic approaches which is possible with animal models as a prelude to application in patient cohorts. It is essential that pediatric neurologists play a major role in taking this period of enlightenment forward so that the outcome for our patients can be dramatically improved.

\section{References}

1 Chen Y, Lu J, Pan H, et al. Association between genetic variation of CACNA1H and childhood absence epilepsy. Ann Neurol 2003; 54(2):239-243

2 Heron SE, Phillips HA, Mulley JC, et al. Genetic variation of CACNA1H in idiopathic generalized epilepsy. Ann Neurol 2004; 55(4):595-596

3 Helbig I, Mefford HC, Sharp AJ, et al. 15q13.3 microdeletions increase risk of idiopathic generalized epilepsy. Nat Genet 2009; 41(2):160-162

4 de Kovel CG, Trucks H, Helbig I, et al. Recurrent microdeletions at $15 q 11.2$ and 16p13.11 predispose to idiopathic generalized epilepsies. Brain 2010;133(Pt 1):23-32

5 Dibbens LM, Mullen S, Helbig I, et al; EPICURE Consortium. Familial and sporadic 15q13.3 microdeletions in idiopathic generalized epilepsy: precedent for disorders with complex inheritance. Hum Mol Genet 2009;18(19):3626-3631

6 Scheffer IE, Grinton BE, Heron SE, et al. PRRT2 phenotypic spectrum includes sporadic and fever-related infantile seizures. Neurology 2012;79(21):2104-2108 
7 Arsov T, Mullen SA, Rogers S, et al. Glucose transporter 1 deficiency in the idiopathic generalized epilepsies. Ann Neurol 2012;72(5): 807-815

8 De Vivo DC, Trifiletti RR, Jacobson RI, Ronen GM, Behmand RA, Harik SI. Defective glucose transport across the blood-brain barrier as a cause of persistent hypoglycorrhachia, seizures, and developmental delay. N Engl J Med 1991;325(10):703-709

9 Steinlein OK, Mulley JC, Propping P, et al. A missense mutation in the neuronal nicotinic acetylcholine receptor alpha 4 subunit is associated with autosomal dominant nocturnal frontal lobe epilepsy. Nat Genet 1995;11(2):201-203

10 Wallace RH, Wang DW, Singh R, et al. Febrile seizures and generalized epilepsy associated with a mutation in the $\mathrm{Na}^{+}$-channel beta1 subunit gene SCN1B. Nat Genet 1998; 19(4):366-370

11 Hildebrand MS, Dahl HHM, Damiano JA, Smith RJH, Scheffer IE, Berkovic SF. Recent advances in the molecular genetics of epilepsy. J Med Genet 2013;50(5):271-279

12 Scheffer IE, Bhatia KP, Lopes-Cendes I, et al. Autosomal dominant frontal epilepsy misdiagnosed as sleep disorder. Lancet 1994; 343(8896):515-517

13 De Fusco M, Becchetti A, Patrignani A, et al. The nicotinic receptor beta 2 subunit is mutant in nocturnal frontal lobe epilepsy. Nat Genet 2000;26(3):275-276

14 Aridon P, Marini C, Di Resta C, et al. Increased sensitivity of the neuronal nicotinic receptor alpha 2 subunit causes familial epilepsy with nocturnal wandering and ictal fear. Am J Hum Genet 2006;79(2):342-350

15 Scheffer IE, Bhatia KP, Lopes-Cendes I, et al. Autosomal dominant nocturnal frontal lobe epilepsy. A distinctive clinical disorder. Brain 1995;118(Pt 1):61-73

16 Derry CP, Heron SE, Phillips F, et al. Severe autosomal dominant nocturnal frontal lobe epilepsy associated with psychiatric disorders and intellectual disability. Epilepsia 2008;49(12):2125-2129

17 Heron SE, Smith KR, Bahlo M, et al. Missense mutations in the sodium-gated potassium channel gene KCNT1 cause severe autosomal dominant nocturnal frontal lobe epilepsy. Nat Genet 2012; 44(11):1188-1190

18 Scheffer IE, Phillips HA, O'Brien CE, et al. Familial partial epilepsy with variable foci: a new partial epilepsy syndrome with suggestion of linkage to chromosome 2. Ann Neurol 1998;44(6):890-899
19 Xiong L, Labuda M, Li DS, et al. Mapping of a gene determining familial partial epilepsy with variable foci to chromosome 22q11q12. Am J Hum Genet 1999;65(6):1698-1710

20 Klein KM, O'Brien TJ, Praveen K, et al. Familial focal epilepsy with variable foci mapped to chromosome 22q12: expansion of the phenotypic spectrum. Epilepsia 2012;53(8):e151-e155

21 Dibbens LM, de Vries B, Donatello S, et al. Mutations in DEPDC5 cause familial focal epilepsy with variable foci. Nat Genet 2013; 45(5):546-551

22 Callenbach PMC, van den Maagdenberg AMJM, Hottenga JJ, et al Familial partial epilepsy with variable foci in a Dutch family: clinical characteristics and confirmation of linkage to chromosome 22q. Epilepsia 2003;44(10):1298-1305

23 Berkovic SF, Serratosa JM, Phillips HA, et al. Familial partial epilepsy with variable foci: clinical features and linkage to chromosome 22q12. Epilepsia 2004;45(9):1054-1060

24 Morales-Corraliza J, Gómez-Garre P, Sanz R, Díaz-Otero F, Gutiérrez-Delicado E, Serratosa JM. Familial partial epilepsy with variable foci: a new family with suggestion of linkage to chromosome 22q12. Epilepsia 2010;51(9):1910-1914

25 Shaw RJ. Cell biology. GATORs take a bite out of mTOR. Science 2013;340(6136):1056-1057

26 Bar-Peled L, Chantranupong L, Cherniack AD, et al. A Tumor suppressor complex with GAP activity for the Rag GTPases that signal amino acid sufficiency to mTORC1. Science 2013; 340(6136):1100-1106

27 Carvill GL, Heavin SB, Yendle SC, et al. Targeted resequencing in epileptic encephalopathies identifies de novo mutations in CHD2 and SYNGAP1. Nat Genet 2013;45(7):825-830

28 Carvill GL, Regan BM, Yendle SC, et al. GRIN2A mutations cause epilepsy-aphasia spectrum disorders. Nat Genet 2013;45(9): 1073-1076

29 Allen AS, Berkovic SF, Cossette P, et al; Epi4K Consortium; Epilepsy Phenome/Genome Project. De novo mutations in epileptic encephalopathies. Nature 2013;501(7466):217-221

30 Depienne C, Trouillard O, Gourfinkel-An I, et al. Mechanisms for variable expressivity of inherited SCN1A mutations causing Dravet syndrome. J Med Genet 2010;47(6):404-410

31 Dibbens LM, Tarpey PS, Hynes K, et al. X-linked protocadherin 19 mutations cause female-limited epilepsy and cognitive impairment. Nat Genet 2008;40(6):776-781 